\title{
Cluster-based fuzzy models for groundwater flow in the unsaturated zone
}

\author{
H. Vernieuwe ${ }^{a}$, N.E.C. Verhoest ${ }^{b, *}$, B. De Baets ${ }^{a}$, R. Hoeben ${ }^{b}$, F.P. De Troch ${ }^{b}$ \\ ${ }^{a}$ Ghent University, Department of Applied Mathematics, Biometrics and Process Control, Coupure links 653, 9000 Gent, Belgium \\ ${ }^{\mathrm{b}}$ Ghent University, Laboratory of Hydrology and Water Management, Coupure links 653, 9000 Gent, Belgium
}

Received 17 November 2005; received in revised form 12 April 2006; accepted 29 June 2006

Available online 27 September 2006

\begin{abstract}
In this paper fuzzy models are used as an alternative to describe groundwater flow in the unsaturated zone. The core of these models consists of a fuzzy rule-based model of the Takagi-Sugeno type. Various fuzzy clustering algorithms are compared in the data-driven identification of these Takagi-Sugeno models. The performance of the resulting fuzzy models is evaluated on the training surface on which they were identified, and on time series measurements of water content values obtained through an experiment carried out by the non-vegetated terrain (NVT) workgroup of the European Microwave Signature Laboratory (EMSL) (see [Mancini M, Hoeben R, Troch PA. Multifrequency radar observations of bare surface soil moisture content: a laboratory experiment. Water Resour Res 1999;35(6):1827-38] and [Hoeben R, Troch PA. Assimilation of active microwave observation data for soil moisture profile estimation. Water Resour Res 2000;36(10):2805-19]). Despite higher errors at the borders of high water content values in the training surface, good results are obtained on the simulation of the time series.
\end{abstract}

(c) 2006 Elsevier Ltd. All rights reserved.

Keywords: Fuzzy clustering algorithms; Fuzzy rule-based models; Takagi-Sugeno models; Unsaturated groundwater flow

\section{Introduction}

The one-dimensional groundwater flow in an unsaturated soil column is generally modelled by means of a partial non-linear differential equation known as the one-dimensional Richards equation. Since this equation can only be solved analytically in some special cases [3], one has to resort to numerical algorithms for approximating its solution. However, an alternative solution to this Richards equation in the form of a fuzzy rule-based model has been proposed by Bárdossy et al. [4-6]. Their

\footnotetext{
* Corresponding author. Tel.: +329 2646138; fax: +3292646236.

E-mail addresses: Hilde.Vernieuwe@UGent.be (H. Vernieuwe), Niko. Verhoest@UGent.be (N.E.C. Verhoest), Bernard.DeBaets@UGent.be (B. De Baets), Rudi.Hoeben@UGent.be (R. Hoeben), Francois.DeTroch@ UGent.be (F.P. De Troch).
}

purpose was to introduce a simpler methodology to calculate the water movement in the soil. Bárdossy et al. [4] used zero-order Takagi-Sugeno rules to model the one-, two- and three-dimensional unsaturated flow. The main advantage of their model is its simplicity and its "small computational requirement". Furthermore, they state that numerical two- and three-dimensional solutions to the Richards equation are scarce and have a high CPU requirement. Although their fuzzy rule-based model gives promising results, some disadvantages can be recognised. Their method is restricted to triangular fuzzy sets, and requires an explicit definition of the support of the antecedent fuzzy sets, indicating that knowledge about the system to model is indispensable. However, this need for knowledge could be bypassed, for instance, by the use of optimisation techniques such as genetic algorithms, demanding an additional effort. Furthermore, their 
method requires a predetermined number of rules. Alternatively, an exhaustive search on the number of rules that yield an accurate fuzzy rule base could be performed.

The objective of this paper is twofold. First, a fuzzy model with similar rules as those described by Bárdossy et al. [4-6] is developed to simulate the one-dimensional unsaturated groundwater flow. The core of our fuzzy groundwater model consists of a first-order Takagi-Sugeno (TS) model: a flexible fuzzy rule-based model that is suitable to describe non-linear systems and that can be identified with a data-driven method. Second, it is investigated which clustering algorithm, including the popular fuzzy $c$ means and Gustafson-Kessel clustering algorithms, used for the identification of the antecedent parts of the fuzzy rules, yields the best TS model. The consequent parameters of the TS rules are computed using the global least squares method [7].

This paper is organised as follows. Section 2 gives an introduction to Takagi-Sugeno models and their identification. The results obtained by the different fuzzy models are evaluated in Section 3. Finally, Section 4 draws the conclusions and gives indications for further research.

\section{Fuzzy models of the Takagi-Sugeno type}

\subsection{Introduction}

The kernel of a fuzzy rule-based model is the fuzzy rule base, containing rules of the form

IF antecedent part THEN consequent part.

These IF-THEN rules describe logical relations between the variables of the system. The rule-based nature of the model allows for a linguistic description of the knowledge, captured in the model (gray-box model), which is an advantage compared to other non-linear modelling techniques such as neural networks (black-box model). The central concept in fuzzy set theory is the fuzzy set or membership function, which numerically represents the degree to which a given element belongs to a fuzzy set. Mathematically speaking, a fuzzy set $A$ on a universe $X$ is defined as a function

$A: X \rightarrow[0,1]: x \mapsto A(x)$,

where $A(x)$ is the degree of membership of $x$ in $A$. The number, position and shape (triangular, trapezoidal,...) of fuzzy sets can be determined from expert knowledge or through data analysis.

The Takagi-Sugeno (TS) model was proposed by Takagi and Sugeno [8]. In order to apply a TS model to a $p$-dimensional input space, in particular if the fuzzy set of the antecedent part $A_{i}$ is a Cartesian product $A_{i}=A_{1, i_{1}} \times \cdots \times A_{p, i_{p}}$, with $i_{1} \in\left\{1, \ldots, n_{1}\right\}, \ldots, i_{p} \in\left\{1, \ldots, n_{p}\right\}$, and $n_{1}, n_{2}, \ldots, n_{p}$ the number of fuzzy sets each input variable is partitioned into, the rule reads:

$$
\begin{aligned}
R_{i} & : \operatorname{IF}\left(X_{1}, \ldots, X_{p}\right) \text { is } A_{i} \text { THEN } Y \\
& =a_{1, i} X_{1}+a_{2, i} X_{2}+\cdots+a_{p, i} X_{p}+b_{i} .
\end{aligned}
$$

For a $p$-dimensional input vector $\mathbf{x}=\left(x_{1}, \ldots, x_{p}\right), A_{i}(\mathbf{x})$ is then usually realised as

$A_{i}(\mathbf{x})=A_{1, i_{1}}\left(x_{1}\right) \cdot A_{2, i_{2}}\left(x_{2}\right) \cdots A_{p, i_{p}}\left(x_{p}\right)$.

When the above type of rules is used, $A_{i}(\mathbf{x})$ is also the degree of fulfilment (DOF) $w_{i}(\mathbf{x})$ of rule $i$. The resulting output value $y$ is then computed as

$y=\frac{\sum_{i=1}^{n} w_{i}(\mathbf{x})\left(a_{1, i} x_{1}+\cdots+a_{p, i} x_{p}+b_{i}\right)}{\sum_{i=1}^{n} w_{i}(\mathbf{x})}$

with $n$ the number of rules. A first-order TS model approximates a non-linear function by means of local linear models, represented in the consequent parts. By computing a weighted average of the individual rule outputs, i.e. the linear functions, the non-linear function can be approximated, and a smooth transition between the consequent functions is established, which is different from an ordinary piecewise linear approximation method [8].

\subsection{Identification of a TS model}

In general, TS models are constructed following a data-driven approach. The identification of TS models consists of the determination of the number of rules, of the antecedent parts and of the parameters of the consequent parts. The input and output variables are assumed to be known beforehand. Different methods can be applied for the identification of the antecedent parts of the rules. Among them are the widely used fuzzy clustering algorithms, from which the obtained fuzzy clusters can serve as multi-dimensional membership functions, or can be projected onto the variable axes in order to obtain one-dimensional membership functions. To each cluster then corresponds a rule. The number of clusters/rules of the TS models in this work was selected using the algorithm ClusterFinder [9] that seeks for the lowest number of clusters/rules which still allows an accurate description of the data set (see Section 2.4).

\subsubsection{Identification of the antecedent parts}

The antecedent parts of the TS models in this paper are identified using fuzzy clustering algorithms: the popular fuzzy $c$-means (FCM) and Gustafson-Kessel (GK) clustering algorithms [10], the simplified Gustafson-Kessel (SGK) algorithm [11], the Gath-Geva (GG) algorithm [12], the simplified Gath-Geva (SGG) algorithm [11] and the modified Gath-Geva (MGG) algorithm [13] are used. All these fuzzy clustering algorithms can be categorised into the group of the objective function-based clustering algorithms, since they all try to minimise the same objective function: 
$J=\sum_{k=1}^{N} \sum_{i=1}^{c} u_{i}\left(\mathbf{z}_{k}\right)^{m} \cdot d\left(\mathbf{z}_{k}, \mathbf{c}_{i}\right)^{2}$.

This objective function can be seen as a generalisation of the least squared error with $N$ the number of data points, $c$ the number of clusters, $d\left(\mathbf{z}_{k}, \mathbf{c}_{i}\right)$ a measure for the distance between data vector $\mathbf{z}_{k}$ and cluster centre $\mathbf{c}_{i}$ and $u_{i}\left(\mathbf{z}_{k}\right) \in[0,1]$ the membership degree of data vector $\mathbf{z}$ to cluster $C_{i}$. The exponent $m \in \mathbb{R}_{>1}$, called fuzzifier, represents an additional parameter. A common choice of the fuzzifier is $m=2[7,11]$ a value that also will be used throughout this paper. All these objective function-based clustering algorithms follow an iterative procedure as shown in Algorithm 1. The iteration starts with a randomly initialised partition matrix for the FCM, GK and SGK clustering algorithms. For the GG, SGG and MGG clustering algorithms, the initial partition matrix is generated by the GK clustering algorithm. The iteration ends when the maximal difference between the partition matrices, which store the membership degrees of the data points to the clusters, of the previous and the current iteration becomes lower than a predetermined tolerance value $\epsilon$ :

$\left\|U^{(l)}-U^{(l-1)}\right\|<\epsilon$.

The major difference between these fuzzy clustering algorithms lies in the calculation of the distance of a data point to a cluster centre. The FCM clustering algorithm uses the Euclidean distance, indicating that data points with the same distance to the cluster centre are situated on a sphere. The GK clustering algorithm uses covariance matrices in calculating the distance between a data point and a cluster centre. Data points with the same distance to the cluster centre are then situated on an ellipsoid. In the GG clustering algorithm, the data are considered to be realisations of normally distributed random variables. This fuzzy clustering algorithm is equivalent to a Gaussian mixture model that expands a probability density function into a sum over $c$ clusters [13]. The distance of a data point to a cluster centre is then calculated as the reciprocal of an unnormalised a posteriori probability or likelihood [11]. The SGK and the SGG clustering algorithms are simplifications of the original GK and GG algorithms in the sense that they only permit axes-parallel clusters, i.e. the covariance matrices have off-diagonal zero elements. The MGG algorithm has been specifically designed to identify TS models, and incorporates the identification of the consequent parameters.

With the objective function-based clustering algorithms, multi-dimensional membership functions are obtained. The membership degree of a data point to the multi-dimensional membership function is calculated as is done in the corresponding clustering algorithm, as a function of the distance to the cluster centre. In order to obtain onedimensional membership functions, the clusters obtained with the objective function-based clustering algorithms are projected onto the variable axes. The projection is mainly performed as it is written in the source code of the Fuzzy Modelling and Identification Toolbox for MATLAB [14] through projection of the partition matrix obtained by each clustering algorithm onto the input variable axes. Just as in the toolbox, the resulting projection is filtered by means of a low-pass filter and exponential membership functions are fitted. In the Fuzzy Modelling and Identification Toolbox, a factor 7 is used in the equation for the exponential membership function. This factor is replaced by a variable factor $\beta$ which was, in our application, increased from 1 to 10 , with steps of size 1 . The exponential membership function is then given by

$A(x)= \begin{cases}\mathrm{e}^{-\frac{\left(x-a_{2}\right)^{2}}{\left(a_{2}-a_{1}\right)^{2}}} & \text { if } x<a_{2}, \\ \mathrm{e}^{-\frac{\left(x-a_{3}\right)^{2}}{\left(a_{4}-a_{3}\right)^{2}}} & \text { if } x>a_{3}, \\ 1 & \text { otherwise. }\end{cases}$

The parameters $a_{2}$ and $a_{3}$ determine the core of the membership function, and $a_{1}$ and $a_{4}$ are the parameters for which the membership degree equals $\mathrm{e}^{-\beta}$. These parameters are obtained by fitting Eq. (7) to the projection of the clusters onto the variable axes.

Algorithm 1: Outline of an objective function-based clustering algorithm

Data: Data set $Z$

Number of clusters $1<c<N$

The weighting exponent $m>1$

The termination tolerance $\epsilon>0$

Result: Cluster centres $\mathbf{c}_{i}$

Other cluster parameters: covariance matrices, a priori probabilities, consequent parameters (for the MGG algorithm), etc.

Initialise partition matrix $U^{(0)}$

while $\left\|U^{(1)}-U^{(1-1)}\right\| \geqslant \epsilon$ do

Compute cluster prototypes $\mathbf{c}_{i}$

Compute other cluster parameters

Compute the distance between data points $\mathbf{z}_{k}$ and cluster centres $\mathbf{c}_{i}$

Update the partition matrix end

\subsubsection{Identification of the consequent parameters}

A TS model consisting of $n$ rules can be seen as a weighted average of $n$ 'regression models'. Therefore, its consequent parameters can be estimated by means of a linear least squares method taking into account the respective weighting parameters $\gamma$, which are functions of the obtained degree of fulfillments. The calculation of the output values by means of a TS model can be expressed in matrix notation:

$O=I \times P$

or 


$$
\begin{aligned}
O=\left[y_{1} \cdots y_{N}\right]^{\mathrm{T}}= & {\left[\begin{array}{ccccccccc}
\gamma_{1,1} x_{1,1} \cdots & \gamma_{1,1} x_{1, p} & \gamma_{1,1} & \cdots & \gamma_{1, n} x_{1,1} & \cdots & \gamma_{1, n} x_{1, p} & \gamma_{1, n} & \\
\vdots & \vdots & \vdots & \vdots & \vdots & \vdots & \vdots & \vdots & \vdots \\
\gamma_{N, 1} x_{N, 1} & \cdots & \gamma_{N, 1} x_{N, p} & \gamma_{N, 1} & \cdots & \gamma_{N, n} x_{N, 1} & \cdots & \gamma_{N, n} x_{N, p} & \gamma_{N, n}
\end{array}\right] } \\
& \times\left[\begin{array}{lllllllllll}
a_{1,1} & a_{1,2} & \cdots & a_{1, p} & b_{1} & \cdots & a_{n, 1} & a_{n, 2} & \cdots & a_{n, p} & b_{n}
\end{array}\right]^{\mathrm{T}}
\end{aligned}
$$

where $\gamma_{k, j}=\frac{w_{j}\left(\mathbf{x}_{k}\right)}{\sum_{i=1}^{n} w_{i}\left(\mathbf{x}_{k}\right)}$ are the normalised membership degrees, with $k=1, \ldots, N ; j=1, \ldots, n$ with $N$ the number of data points and $n$ the number of rules. Using $\gamma_{k, j}$ in $I$, the weighting of the rules is taken into account, and a minimal global prediction error is obtained. The parameters $P$ are then estimated by

$P=\left[I^{\mathrm{T}} I\right]^{-1} I^{\mathrm{T}} O$.

This method is also referred to as the global least squares method [7]. Alternatively, the recursive least squares method [15] which bypasses the problem of singularity of $\left[I^{\mathrm{T}} I\right]$ can be used.

\subsection{Performance indices for Takagi-Sugeno models}

The accuracy of the identified TS models is evaluated using two performance indices. The first index is the Nash and Sutcliffe (NS) performance measure [16]:

$\mathrm{NS}=1-\frac{\sum_{k=1}^{N}\left(y_{\mathrm{m}}(k)-y(k)\right)^{2}}{\sum_{k=1}^{N}(y(k)-\bar{y})^{2}}$,

with $N$ the number of data points, and $y_{\mathrm{m}}$ the modelled and $y$ the observed output. $\bar{y}$ denotes the mean of the observed values. The optimal value of NS is 1 , meaning a perfect match of the model. A value of zero indicates that the model predictions are as good as that of a 'no-knowledge' model continuously simulating the mean of the observed signal. Negative values indicate that the model is performing worse than this 'no-knowledge' model [17]. The second performance index is the root mean square error (RMSE):

$\mathrm{RMSE}=\sqrt{\frac{\sum_{k=1}^{N}\left(y(k)-y_{\mathrm{m}}(k)\right)^{2}}{N}}$.

The RMSE takes positive values with an optimal value of 0 , indicating a perfect match of the model.

\subsection{ClusterFinder}

Evaluating the values of the performance indices for models with an increasing number of rules, e.g. models with 2-64 clusters/rules, confirmed an obvious relationship between the accuracy and the complexity of the models: the complexer the model, the more accurate its performance. It was also observed that adding an extra cluster strongly improved the values of the NS- or RMSE-index for a low number of clusters, whereas the improvement due to an additional cluster became less apparent for a higher number of clusters. Hadjili and Wertz [18] point out that three approaches are commonly used to determine the appropriate number of clusters. In the first approach, one starts with a small number of clusters, and subsequently adds more clusters based on the prediction error between the model and the system. The second approach starts with a large number of clusters and subsequently removes redundant clusters based on e.g. a similarity measure between clusters, while the third aims at acquiring "good partitions". Throughout the literature, several cluster validity measures (see [12,19-21] among others) have been proposed to obtain these "good partitions". The criteria for the definition of an "optimal partition" of the data into subgroups are based on three requirements [12]: a clear separation between the resulting clusters, a minimal volume of the clusters, and a maximum number of data points concentrated in the vicinity of the cluster centre. However, in this paper, an optimal model rather than an optimal partition is sought for. Therefore, the number of clusters is determined based on the quality of the prediction obtained with the resulting model. Vernieuwe et al. [9] developed the algorithm ClusterFinder to search for the number of clusters that yield a model with an acceptable accuracy and complexity. For the models identified in this paper, ClusterFinder starts with the identification of models with 64 clusters. The optimal number of clusters is then determined by means of a recursive algorithm in which the performance of the candidate model is compared with the performance of the model with 64 clusters. The optimal model is found as the model with the lowest possible number of clusters for which the value of the performance index does not differ more than a predefined margin $\alpha$ from the value of the performance index for the model with 64 clusters. In order to account for the influence of the initialisation on the clustering, 30 repetitions were performed of which the best model was retained and used for ClusterFinder. In this paper, the value of $\alpha$ was set at 0.02 and ClusterFinder was applied to the NS values.

\section{Fuzzy models for 1D unsaturated groundwater flow}

\subsection{Structure and identification of the fuzzy groundwater models}

The water movement in a one-dimensional, isotropic soil matrix is described by the one-dimensional Richards equation $[3,22]$ : 
$\frac{\partial \theta}{\partial t}=-\frac{\partial}{\partial z}\left(K \frac{\partial \psi}{\partial \theta} \frac{\partial \theta}{\partial z}+K\right)$

where $\theta$ is the soil water content $[-], t$ is the time $[\mathrm{T}], z$ is the gravity head expressing the elevation [L] of a point with respect to the soil surface for which $z=0$, defined positive upward, $K$ is the hydraulic conductivity of the soil $[\mathrm{L} / \mathrm{T}]$ and $\psi$ is referred to as the matric head [L], which both vary with the soil water content. The matric head $\psi$ and the soil water content $\theta$ are linked through the van Genuchten equation [23]:

$\theta(\psi)=\theta_{\mathrm{r}}+\left(\theta_{\mathrm{s}}-\theta_{\mathrm{r}}\right)\left(\frac{1}{1+\left(\alpha_{\mathrm{vg}} \psi\right)^{n_{\mathrm{vg}}}}\right)^{m_{\mathrm{vg}}}$,

where $\alpha_{\mathrm{vg}}, n_{\mathrm{vg}}, m_{\mathrm{vg}}$ are soil-related parameters (with $m=1-1 / n), \theta_{\mathrm{s}}$ is the saturated water content and $\theta_{\mathrm{r}}$ is the residual water content of the soil.

In this paper, the solution of the Richards equation is approximated by means of a first-order TS model with rules of the following form:

$R_{i}: \operatorname{IF}\left[\left(\frac{\theta}{\theta_{\mathrm{s}}}\right)_{\mathrm{u}},\left(\frac{\theta}{\theta_{\mathrm{s}}}\right)_{1}\right]$ IS $A_{i}$ THEN
$q_{v}=a_{i}\left(\frac{\theta}{\theta_{\mathrm{s}}}\right)_{\mathrm{u}}+b_{i}\left(\frac{\theta}{\theta_{\mathrm{s}}}\right)_{1}+c_{i}$,

with $A_{i}$ a multi-dimensional membership function and $a_{i}, b_{i}$ and $c_{i}$ the consequent parameters. The subscripts $\mathrm{u}$ and 1 refer to respectively the upper and lower soil cell between which the flux is calculated. If one-dimensional membership functions are used, $A_{i}$ represents the cartesian product $A_{1, i} \times A_{2, i}$. The TS model, either with one- or multidimensional membership functions, calculates the flux $q_{v}$ between two adjacent soil cells with relative water content $\left(\frac{\theta}{\theta_{\mathrm{s}}}\right)_{\mathrm{u}}$ and $\left(\frac{\theta}{\theta_{\mathrm{s}}}\right)_{1}$.

The fuzzy groundwater model, incorporating the TS model, updates the water content with the simulated fluxes. Given an initial soil profile of water contents, the TS model calculates the incoming $q_{v \text {,in }}$ and outgoing $q_{v \text {,out }}$ fluxes for each soil cell with thickness $\Delta z$. Using the initial water content $\theta_{\text {old }}$ and the newly calculated fluxes at time $t$, the water content $\theta_{\text {new }}$ at time $t+\Delta t$ is calculated using the continuity equation:

$\theta_{\text {new }}=\left(\theta_{\text {old }} \Delta z+q_{v \text {,in }} \Delta t-q_{v, \text { out }} \Delta t\right) / \Delta z$.

The newly obtained water contents are given as input to the TS model to calculate new fluxes at time $t+\Delta t$ and the process restarts. Evaporation or rainfall values are used to set the boundary conditions at the top of the soil profile. A zero flux or constant water content are the boundary conditions that can be imposed at the bottom of the profile. During rainfall, the infiltration rate equals the rainfall intensity, until the upper soil cell is saturated. In this case, the water content in the upper soil cell equals $\theta_{\mathrm{s}}$. If this occurs, we consider the water excess to be transported through runoff, i.e. ponding of water on the soil profile is not accounted for. A backstepping procedure is applied to determine the time when the soil cell reached saturation. From that moment on, water can infiltrate at a pace equal to the hydraulic conductivity. If the upper soil cell reaches a water content lower than or equal to the water content corresponding to $\mathrm{pF}=3$ during the interstorm period, and an evaporation rate $Q_{\mathrm{a}}$ is imposed, the amount of water $Q_{\mathrm{s}}$ the soil is able to supply decreases linearly as follows (see [2]):

$Q_{\mathrm{s}}=Q_{\mathrm{a}} \frac{\theta-\theta_{\mathrm{WP}}}{\theta_{\mathrm{pF}_{3}}-\theta_{\mathrm{WP}}}$,

with $\theta_{\mathrm{pF}_{3}}$ and $\theta_{\mathrm{WP}}$ the water content at $\mathrm{pF}=3$ and wilting point, respectively.

The number of rules and the corresponding antecedent and consequent parts of these rules were identified on a training data set, consisting of the relative water contents for the upper and lower soil cells and the flux between those soil cells (see Fig. 1). This training data set was synthetically generated using a discretisation of the Darcy equation:

$q=\sqrt{K_{\mathrm{u}} K_{1}}\left(\frac{h_{\mathrm{u}}-h_{1}+\Delta z}{\Delta z}\right)$

with $K_{\mathrm{u}}$ and $K_{1}$ the hydraulic conductivity [L/T], in accordance with the $h$-value [L], of the upper and lower soil cell, respectively, and $\Delta z$, the discretisation step [L]. With this equation, only the vertical water movement is considered and the $z$-axis is chosen positive upward while the positive flux points downward.

An initial data set was generated for a sandy loam soil with the van Genuchten parameters given in Table 1. The relative water content $\theta / \theta_{\mathrm{s}}$ of both upper and lower layer were discretised with steps of size 0.0025 and all possible moisture combinations between both layers resulted in a data set of 154449 data points. In view of the CPU time that would be required to cluster this data set, the number of data points had to be reduced. Therefore a training data set of 10000 data points was randomly selected and the remaining data points were used as validation data set. Based on this validation set, models will be retained and

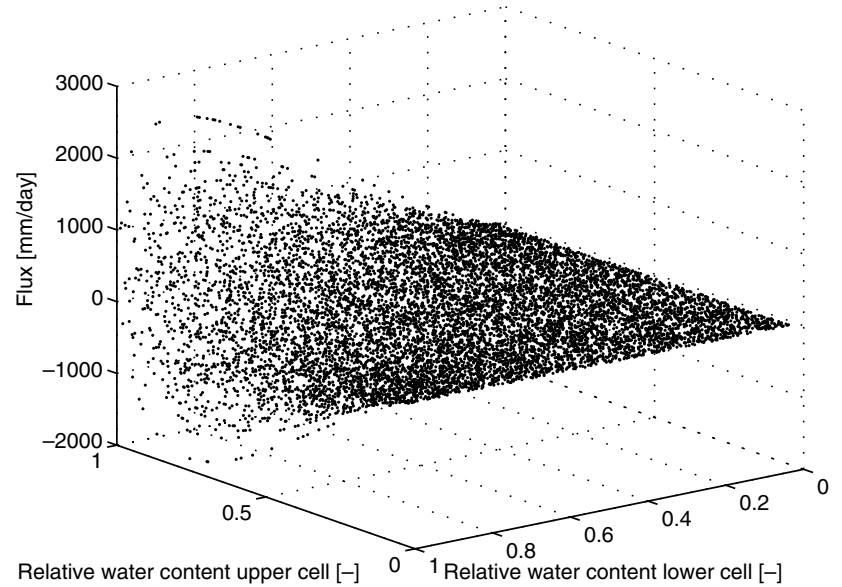

Fig. 1. Training data set. 
Table 1

The van Genuchten parameters for the EMSL data set [2]

\begin{tabular}{lll}
\hline$\theta_{\mathrm{s}}$ & {$[-]$} & 0.55 \\
$\theta_{\mathrm{r}}$ & {$[-]$} & 0.01 \\
$K_{\mathrm{s}}$ & {$[\mathrm{cm} / \mathrm{h}]$} & 5 \\
$\alpha_{\mathrm{vg}}$ & {$[1 / \mathrm{cm}]$} & 0.048 \\
$n_{\mathrm{vg}}$ & {$[-]$} & 1.5632 \\
$m_{\mathrm{vg}}$ & {$[-]$} & 0.3603 \\
\hline
\end{tabular}

will be further evaluated on time series of moisture contents obtained through wetting and drying experiments set up by the non-vegetated terrain (NVT) workgroup of the European Microwave Signature Laboratory (EMSL), Joint Research Centre of the European Community in Ispra (Italy) [1,2]. In this experiment, a cylindric container with diameter and height of respectively $2 \mathrm{~m}$ and $0.4 \mathrm{~m}$ was filled with a sandy loam soil. The moisture conditions of the soil were monitored by means of time domain reflectometry (TDR) probes. These TDR probes were placed along three vertical transects at different distances from the centre of the cylinder. The probes were positioned at depths of $2.5 \mathrm{~cm}, 5 \mathrm{~cm}, 10 \mathrm{~cm}, 15 \mathrm{~cm}$ and $25 \mathrm{~cm}$. The cylinder was subjected to wetting and drying phases, during which the soil moisture profiles were monitored every 10 min. Given the depths of the TDR probes, the spatial discretisation in the generation of the training set was chosen to be $2.5 \mathrm{~cm}$. Fig. 1 shows the training data set. By means of the methods described in Section 2.2, and based on this training data set, the fuzzy rule-based groundwater models can be identified.

\subsection{Evaluation of the fuzzy groundwater models}

The evaluation of the fuzzy groundwater models is performed on the training surface as well as on two time series obtained for one of the transects in the EMSL experiment. For these time series, the evaluation of the fuzzy groundwater models is performed by means of a comparison with the measurements and with the results obtained with the numerical model of Hoeben and Troch [2] for the same initial and boundary conditions. The boundary conditions at the upper soil cell are given by rainfall and evaporation during the experiment and remain the same throughout the simulations. Two initial conditions were possible: the first consists of a partition of the soil profile in homogeneous blocks of the same water content given by the initial measurements with the TDR probes in the experiment, the second uses the measured water contents at the depths of the TDR probes and a linear interpolated water content at depths in between two measurements. Since the TS models were identified on a training surface obtained through the solution of the Darcy equation for a discretisation of the entire domain of water contents, more attention was given to the comparison of the results of both models than to the comparison of the results with the measurements.

\subsubsection{Takagi-Sugeno models with multi-dimensional membership functions}

The obtained numbers of clusters with the corresponding performance on the training data set are listed in Table 2. As ClusterFinder searches for the optimal number of clusters, based on the performance values of the models with 64 clusters, the values of the performance indices for these models are listed as well. This table shows that the worst values both in terms of NS and RMSE are obtained with the FCM algorithm. The values of the performance indices corresponding to the other fuzzy clustering algorithms are rather similar. The best obtained values for the optimal number of clusters are given by the GG algorithm. Furthermore, it can be seen that the values of the NS-index differ just less than 0.02 from the values for the corresponding models with 64 clusters and that, given a value of 0.02 for the margin $\alpha$, different numbers of clusters for the different models can be obtained.

Fig. 2 illustrates the error surface on the training data set for the GG model. The error surface exhibits large errors at the borders corresponding to high water content. The presence of those large errors is due to the steepness of the slope of the training surface in these areas. The error surfaces of more complex models, e.g. for a model with 64 clusters, show that these errors are considerably reduced with respect to those of the optimal models (data not

Table 2

Number of clusters obtained with ClusterFinder for the different fuzzy clustering algorithms and the performance of the corresponding models

\begin{tabular}{llllll}
\hline $\begin{array}{l}\text { Clustering } \\
\text { method }\end{array}$ & \#Clusters & $\begin{array}{l}\mathrm{NS} \\
{[-]}\end{array}$ & $\begin{array}{l}\mathrm{RMSE} \\
{[\mathrm{mm} / \mathrm{day}]}\end{array}$ & $\begin{array}{l}\mathrm{NS}_{64} \\
{[-]}\end{array}$ & $\begin{array}{l}\mathrm{RMSE}_{64} \\
{[\mathrm{~mm} / \mathrm{day}]}\end{array}$ \\
\hline FCM & 19 & 0.9458 & 73.3523 & 0.9642 & 59.5813 \\
GK & 16 & 0.9794 & 45.1877 & 0.9989 & 10.4480 \\
SGK & 17 & 0.9735 & 51.2496 & 0.9921 & 27.9158 \\
GG & 22 & $\mathbf{0 . 9 8 0 9}$ & $\mathbf{4 3 . 5 1 8 8}$ & 0.9987 & 11.3245 \\
SGG & 33 & 0.9791 & 45.5201 & 0.9964 & 18.9872 \\
MGG & 37 & 0.9768 & 48.0146 & 0.9953 & 21.6053 \\
\hline
\end{tabular}

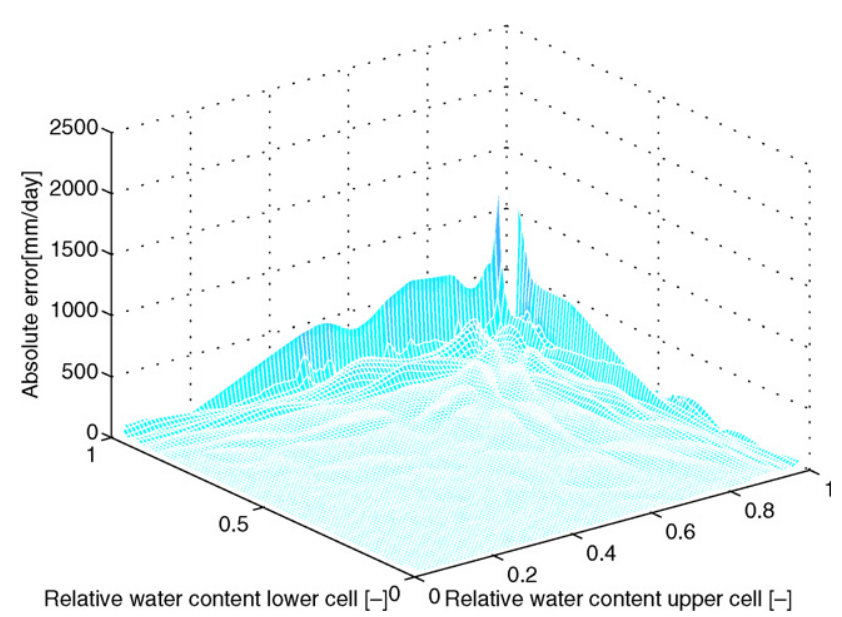

Fig. 2. Errors on the training data set for the model with 22 rules identified with the Gath-Geva clustering algorithm. 
shown). A more complex model smoothens the error surface and is able to reduce the errors at the borders of high water content. Still, using a less complex model seems a reasonable choice to obtain a sufficiently high performance since the transition between water contents in a soil profile is mainly gradual. The location of the water content combinations on the training surface, during simulation, will mainly be around the diagonal from the $(0,0)$ corner to the $(1,1)$ corner (see further). For all models, low (or almost no) errors are found in the regions with low or moderate water content.

The models of which the obtained number of clusters and performances are listed in Table 2 were evaluated on the two time series of the EMSL experiment. The obtained results, expressed as mean absolute errors in water contents, are listed in Tables 3 and 4. The results of the fuzzy models are compared with the measurements as well as with the numerical model of Hoeben and Troch [2] applied under the same boundary and initial conditions.

Generally, poor results are obtained for the FCM and SGK models on both time series. The GK model also shows some poor results on the first time series given an initial homogeneous partition of water contents and given initial interpolated water contents with a constant water content at the bottom of the profile.

The models identified with the GG algorithm and its variants approximate the results of the numerical model best for both time series. Among these models, the MGG model yields the best values. Some dependence on the different initial conditions can be observed for the SGK, SGG and MGG models. A dependence on the different boundary conditions at the bottom of the profile can be noticed for the SGK model. Based on Tables 3 and 4 on the one hand and Table 2 on the other hand, the models can be ranked in a different order of performance. This is due to the fact that water contents reached during the evaluation

Table 3

Mean absolute errors with the measurements and the numerical model for the different fuzzy models for the first time series

\begin{tabular}{lcclll}
\hline Clustering algorithm & \multicolumn{2}{l}{ Interpolation } & & \multicolumn{2}{l}{$\begin{array}{l}\text { Homogeneous } \\
\text { blocks }\end{array}$} \\
\cline { 2 - 3 } & ZF & CM & & ZF & CM \\
\hline Comparison with measurements & & & & \\
FCM & 0.0908 & 0.0980 & & 0.0859 & 0.1004 \\
GK & 0.0343 & 0.0334 & & 0.0494 & 0.0485 \\
SGK & 0.0667 & 0.0587 & & 0.0727 & 0.0595 \\
GG & 0.0242 & 0.0244 & & 0.0286 & 0.0280 \\
SGG & 0.0223 & 0.0223 & & 0.0238 & 0.0238 \\
MGG & 0.0227 & 0.0226 & & 0.0236 & 0.0236 \\
Numerical model & 0.0210 & 0.0210 & & 0.0202 & 0.0202 \\
Comparison with numerical model & & & & \\
FCM & 0.1022 & 0.1077 & & 0.0946 & 0.1097 \\
GK & 0.0320 & 0.0525 & & 0.0924 & 0.0916 \\
SGK & 0.0625 & 0.0479 & & 0.0660 & 0.0484 \\
GG & 0.0152 & 0.0154 & & 0.0131 & 0.0124 \\
SGG & 0.0087 & 0.0087 & & 0.0081 & 0.0081 \\
MGG & $\mathbf{0 . 0 0 4 3}$ & $\mathbf{0 . 0 0 4 4}$ & $\mathbf{0 . 0 0 3 3}$ & $\mathbf{0 . 0 0 3 3}$ \\
\hline
\end{tabular}

Table 4

Mean absolute errors with the measurements and the numerical model for the different fuzzy models for the second time series

\begin{tabular}{lcclll}
\hline Clustering algorithm & \multicolumn{2}{l}{ Interpolation } & & \multicolumn{2}{l}{$\begin{array}{l}\text { Homogeneous } \\
\text { blocks }\end{array}$} \\
\cline { 2 - 3 } & ZF & CM & & ZF & CM \\
\hline Comparison with measurements & & & \\
FCM & 0.0438 & 0.0853 & & 0.0401 & 0.0818 \\
GK & 0.0271 & 0.0270 & & 0.0272 & 0.0272 \\
SGK & 0.0626 & 0.0899 & & 0.0602 & 0.0857 \\
GG & 0.0298 & 0.0367 & & 0.0268 & 0.0341 \\
SGG & 0.0248 & 0.0248 & & 0.0238 & 0.0238 \\
MGG & 0.0238 & 0.0241 & & 0.0219 & 0.0222 \\
Numerical model & 0.0206 & 0.0205 & & 0.0217 & 0.0216 \\
Comparison with numerical model & & & & \\
FCM & 0.0365 & 0.0780 & & 0.0346 & 0.0762 \\
GK & 0.0214 & 0.0234 & & 0.0203 & 0.0226 \\
SGK & 0.0554 & 0.0789 & & 0.0547 & 0.0779 \\
GG & 0.0149 & 0.0208 & & 0.0115 & 0.0178 \\
SGG & 0.0100 & 0.0101 & & 0.0099 & 0.0099 \\
MGG & $\mathbf{0 . 0 0 4 2}$ & $\mathbf{0 . 0 0 4 2}$ & $\mathbf{0 . 0 0 4 1}$ & $\mathbf{0 . 0 0 4 2}$ \\
\hline
\end{tabular}

on these two time series only cover a part of the entire domain of possible water contents while the performance indices of Table 2 on the contrary, are calculated based on the complete domain. Figs. 3 and 4 show the simulation results for the MGG model. The simulations performed with the numerical model are plotted as well. The initial and the lower boundary conditions used consist of homogeneous blocks of water contents and a zero flux at the bottom of the profile. From these figures, it follows that a good agreement between the numerical and the fuzzy rule-based model is obtained. Both models show the same behaviour.

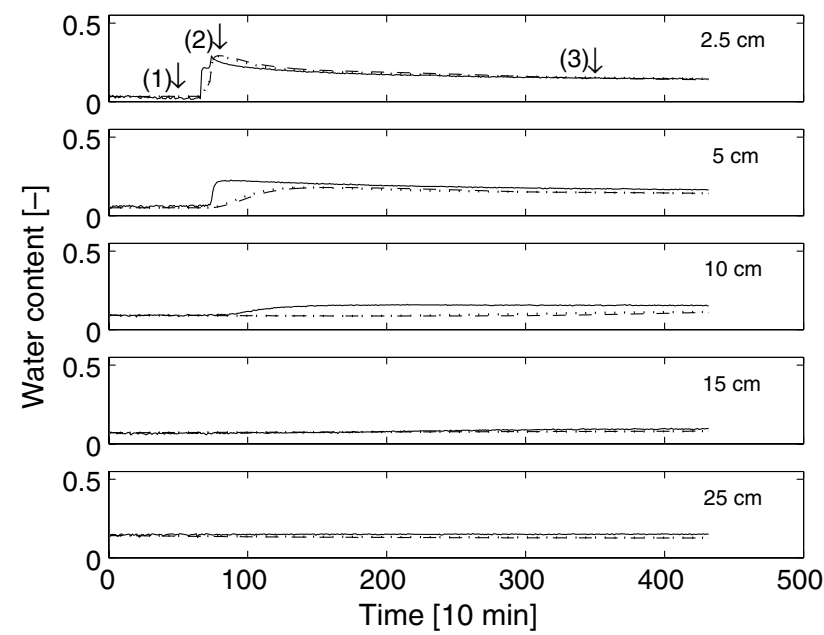

Fig. 3. Measurements (solid lines) and simulation results of the MGG model (dashed lines) and the numerical model (dotted lines) for the first time series. The initial and lower boundary conditions used consist of homogeneous blocks of water content and a zero flux at the bottom of the profile. The time steps upon which moisture profiles are captured are indicated. 


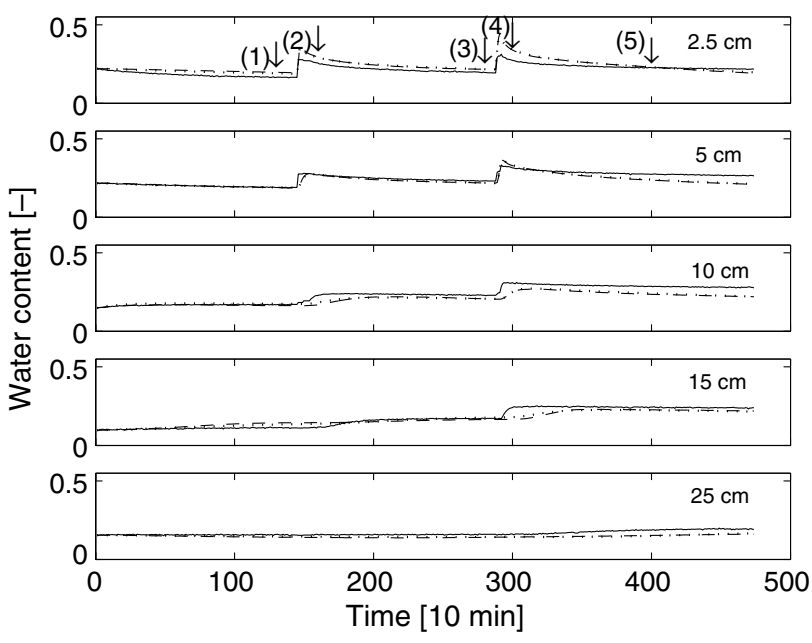

Fig. 4. Measurements (solid lines) and simulation results of the MGG model (dashed lines) and the numerical model (dotted lines) for the second time series. The initial and lower boundary conditions used consist of homogeneous blocks of water content and a zero flux at the bottom of the profile. The time steps upon which moisture profiles are captured are indicated.

The mean absolute errors listed in Tables 3 and 4 only compare the results at measured depths. The behaviour of the models throughout the soil profile is therefore not known. For this reason, moisture profiles at certain time steps of the time series (see Figs. 5 and 6) are plotted for the MGG model and the numerical model. The time steps upon which these profiles are captured, are indicated in Figs. 3 and 4 with the numbers corresponding to those of Figs. 5 and 6 . The initial and the lower boundary conditions used to obtain these profiles consist of homogeneous blocks of water contents and a zero flux at the bottom of the profile. Generally, a very good agreement between both results is observed. At the bottom of the profile, the results of the fuzzy model diverge little from the results of the numerical model.

As an example, water contents obtained through simulation of the second time series are displayed in Fig. 7, together with the contours of the error surface obtained for the MGG model. The largest part of the obtained water contents are located in the area with errors smaller than $10 \mathrm{~mm} /$ day. The remaining part, however, exhibits larger errors. A few obtained combinations of water contents are situated in these regions of higher errors. The errors made by the fuzzy model at a certain time step cause a miscalculation of the water contents for the next time step, influencing the further simulations of the fluxes and water contents throughout the remaining simulation time and the soil profile.

The water content values in the time series of the EMSL experiment are located in the region of the error surface with low errors. The influence of the larger errors observed in the error surfaces on the simulation of the time series is
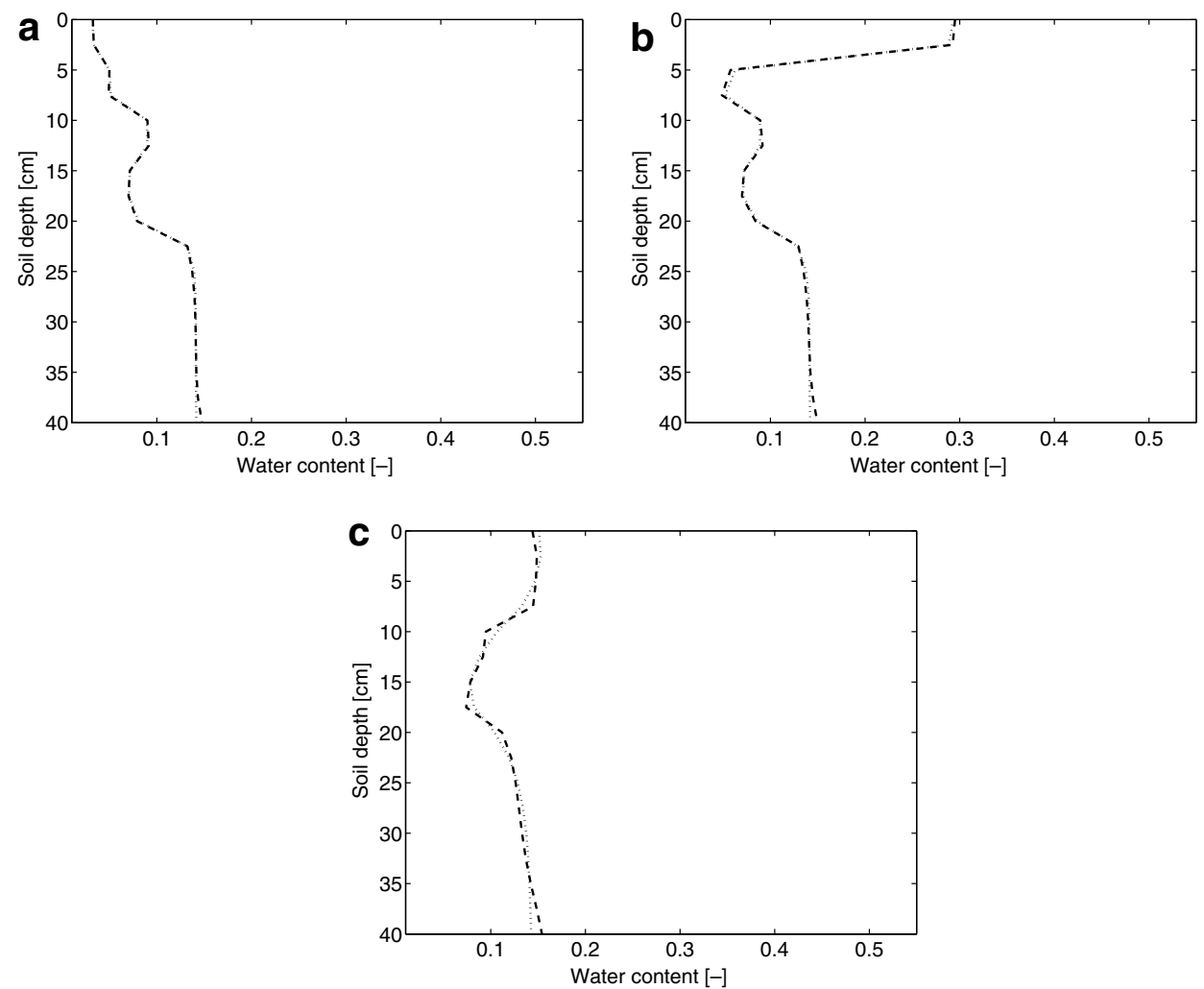

Fig. 5. Moisture profiles for the first time series. Results of the MGG model (dashed lines) and the numerical model (dotted lines). The initial and lower boundary conditions used consist of homogeneous blocks of water content and a zero flux at the bottom of the profile. The time steps upon which these profiles are captured are indicated in Fig. 3. 

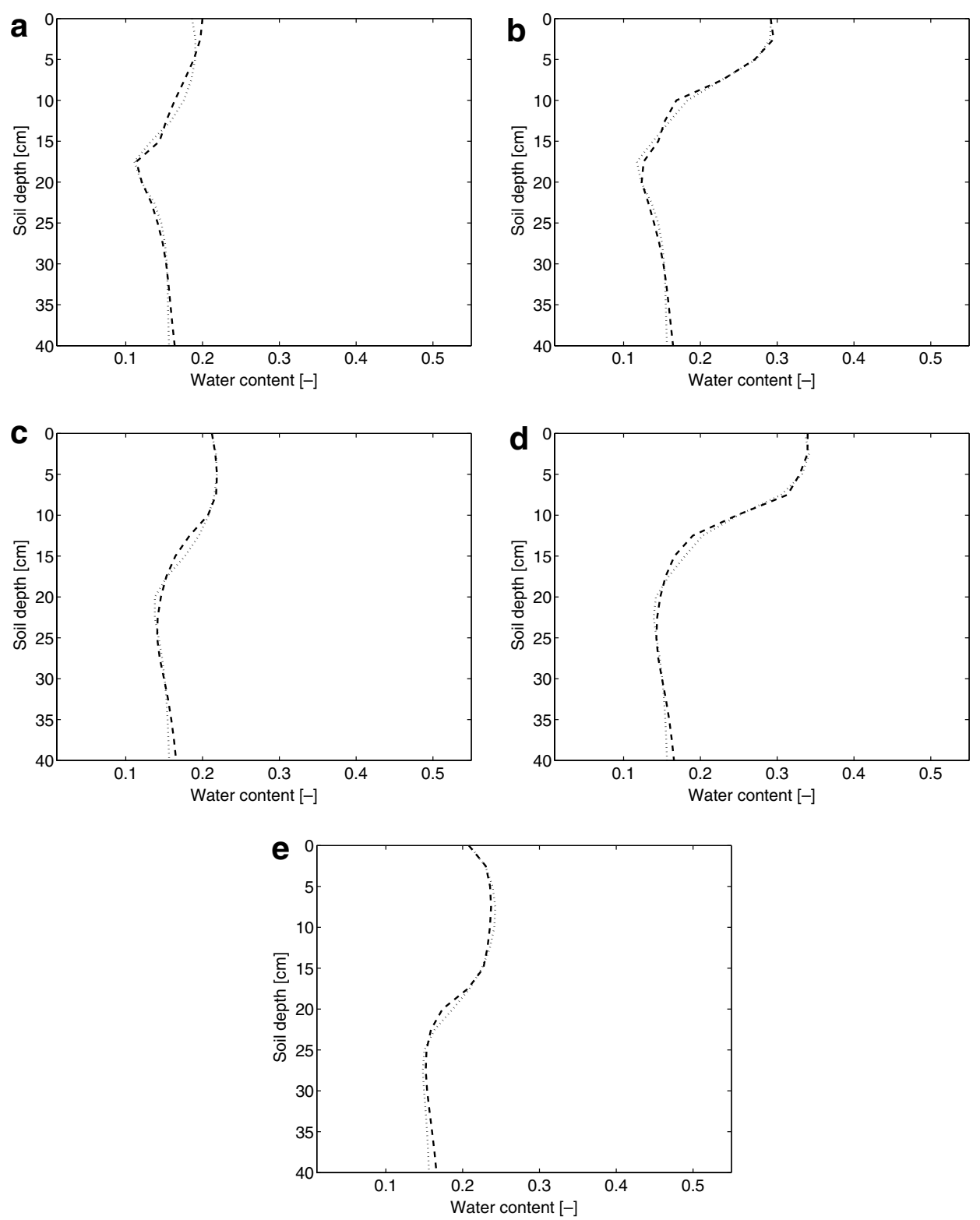

Fig. 6. Moisture profiles for the second time series. Results of the MGG model (dashed lines) and the numerical model (dotted lines). The initial and lower boundary conditions used consist of homogeneous blocks of water content and a zero flux at the bottom of the profile. The time steps upon which these profiles are captured are indicated in Fig. 4.

evaluated with additional tests. The initial water contents of the two original time series were incremented by 0.1 , 0.2 or 0.3 . Since the best mean absolute errors were obtained with the GG model, this model was employed to undergo the additional tests. The results are compared with the results of the numerical model under the same initial and boundary conditions (see Tables 5 and 6). The results show that an increasing initial water content leads to an increasing error. Furthermore, it is observed that when higher water contents are reached, the same errors are obtained for different implementations of initial conditions, i.e. interpolation or homogeneous blocks of water contents, but with the same lower boundary condition. When the original initial water contents are, for instance, increased with 0.3 , an error of 0.016 is obtained when the lower boundary condition is a constant water content for both interpolation and homogeneous blocks of water contents, and an error of ca. 0.014 is obtained when the lower boundary condition is a zero flux, again for both implementations of the initial conditions. This is probably due to the fact that, in the case of a very wet soil profile, the lower boundary conditions, in this case a constant water content or a zero imposed flux, have a larger influence on the water contents reached by the model. Fig. 8 shows the position of the water content combinations on a contour plot of the error surface of the MGG model. These water content combinations were obtained for interpolated initial water contents augmented by 0.3 and a constant zero 


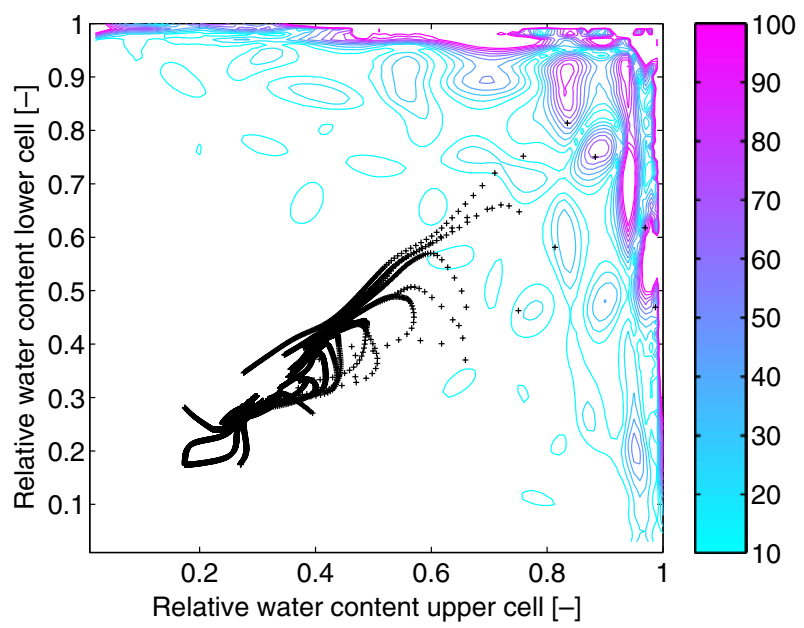

Fig. 7. Contour plot of the absolute errors on the training data set for the MGG model (contour levels from 10 to $100 \mathrm{~mm} /$ day are indicated). The water content combinations obtained for the second time series (interpolation and ZF) are indicated.

flux and a constant water content as respective lower boundary conditions. These water contents are the results of the performance of the additional test on the second time series. The water contents reached by the model on which a constant zero flux is imposed as lower boundary condition are more situated in the region of water contents nearby saturation whereas the water contents reached by the model on which a constant water content is imposed as lower boundary condition are more situated in the region of moderate to higher water contents.

\subsubsection{Takagi-Sugeno models with projected membership functions}

A second way of identifying TS models was performed by projecting the multi-dimensional clusters obtained by the different fuzzy clustering algorithms onto the input variable axes. In this way, one-dimensional exponential membership functions as in Eq. (7) were obtained. The degree of fulfilment of a rule is then obtained by multiplying the membership degrees of the considered data point to the antecedent fuzzy sets (see Eq. (3)). The projection proce-

dure of the toolbox of Babuška, as explained in Section 2.2 is used. Exponential membership functions are fitted to the projections. In the toolbox, a factor 7 is used in the equation for the exponential membership function. This factor is replaced by a variable factor $\beta$ which was, for these applications, increased from 1 to 10 , with steps of size 1 .

Concerning the values of the performance indices, similar results as for the models with multi-dimensional membership functions were obtained. A remarkably better performance for models having their origin in the FCM clustering algorithm was observed. Best results were obtained for the model with 26 rules, obtained with the

Table 5

Mean absolute errors with the numerical model for the additional tests on the first time series

\begin{tabular}{llllll}
\hline Clustering algorithm & \multicolumn{2}{l}{ Interpolation } & & \multicolumn{2}{l}{$\begin{array}{l}\text { Homogeneous } \\
\text { blocks }\end{array}$} \\
& $\mathrm{ZF}$ & $\mathrm{CW}$ & & $\mathrm{ZF}$ & $\mathrm{CW}$ \\
\hline $\begin{array}{l}\text { Initial condition }+0.1 \\
\text { MGG }\end{array}$ & 0.0020 & 0.0032 & & 0.0034 & 0.0028 \\
$\begin{array}{l}\text { Initial condition }+0.2 \\
\text { MGG }\end{array}$ & 0.0073 & 0.0135 & & 0.0073 & 0.0126 \\
$\begin{array}{l}\text { Initial condition }+0.3 \\
\text { MGG }\end{array}$ & 0.0147 & 0.0164 & 0.0136 & 0.0160 \\
\hline
\end{tabular}

Table 6

Mean absolute errors with the numerical model for the additional tests on the second time series

\begin{tabular}{llllll}
\hline Clustering algorithm & \multicolumn{2}{l}{ Interpolation } & & \multicolumn{2}{l}{$\begin{array}{l}\text { Homogeneous } \\
\text { blocks }\end{array}$} \\
\cline { 2 - 3 } & $\mathrm{ZF}$ & $\mathrm{CW}$ & & $\mathrm{ZF}$ & $\mathrm{CW}$ \\
\hline $\begin{array}{l}\text { Initial condition }+0.1 \\
\mathrm{MGG}\end{array}$ & 0.0037 & 0.0041 & & 0.0038 & 0.0041 \\
$\begin{array}{l}\text { Initial condition }+0.2 \\
\mathrm{MGG}\end{array}$ & 0.0106 & 0.0178 & & 0.0103 & 0.0174 \\
$\begin{array}{l}\text { Initial condition }+0.3 \\
\mathrm{MGG}\end{array}$ & 0.0380 & 0.0384 & 0.0382 & 0.0405 \\
\hline
\end{tabular}
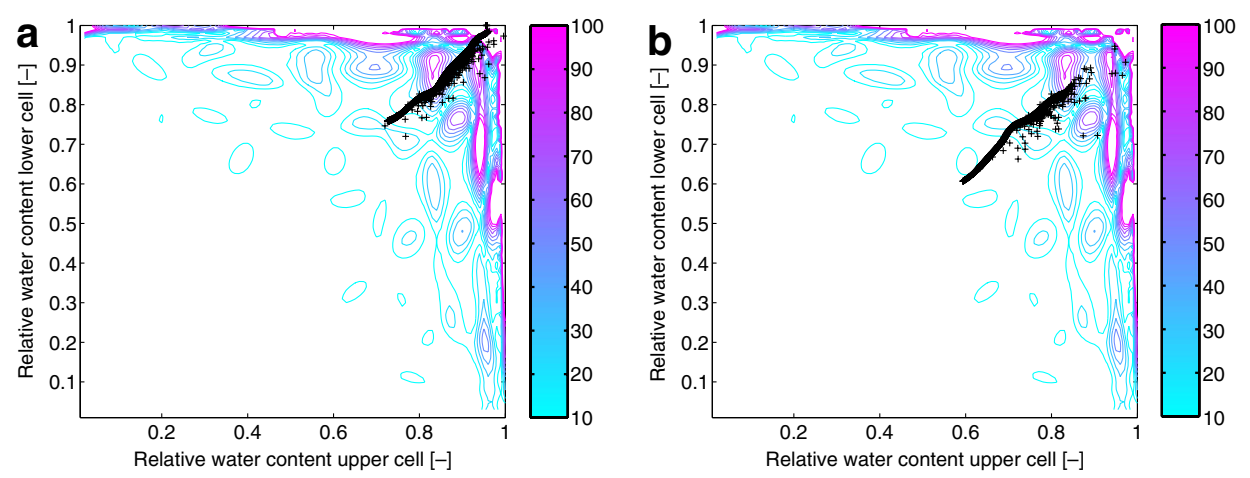

Fig. 8. Combination of water contents reached by the MGG model on the additional test $(+0.3)$ on the second time series for initial interpolated water contents and a constant zero flux (a) and a constant water content (b) as respective lower boundary conditions. 
MGG algorithm with $\beta=2$, namely $\mathrm{NS}=0.9813$ and RMSE $=43.1025 \mathrm{~mm} /$ day.

The resulting models were used to simulate the two time series of the EMSL experiment. For each obtained number of rules, 10 models were evaluated, i.e. $\beta$-values from 1 to 10 were considered. The best obtained mean absolute errors in water content between the simulations of the fuzzy rule-based and the numerical model for the first time series were obtained for the SGG model and the MGG model with mean absolute errors of 0.0071 for the initial condition of interpolated water contents for both boundary conditions for the SGG model, and with mean absolute errors of 0.0081 and 0.0082 for the initial conditions of homogeneous blocks of water content for the MGG model. The $\beta$-value for which these results are obtained are 3 and $2-6,8-10$ for the SGG model, and 7 for the MGG model. Concerning the simulations on the second time series, the best results are obtained for the MGG model with mean absolute errors of 0.0082 and 0.0079 for initial interpolated water contents and homogeneous blocks of water content respectively. The $\beta$-value for which these results are obtained range from 1 to 10 . In general, the obtained results for other $\beta$-values are quite similar. Larger differences, ranging from approximately 0.01 to approximately 0.02 , between the results of different $\beta$-values are found for the FCM, GK, GG and SGK models. In most cases, the best simulations on the time series are obtained for different values of $\beta$ than those obtained on the training surface. This implies that models with different $\beta$-values perform better in the area reached by the water contents in the time series, than models that generally perform best for the entire domain. Furthermore, the value of $\beta$ can change with different initial and/or boundary conditions, since the area reached by the water contents is likely to be influenced by these conditions. A straightforward conclusion concerning the optimal value of $\beta$-value can therefore not be made.

The obtained differences for all simulation results (data not shown) stay within a range of approximately $4 \%$ water content, which is fairly acceptable. Concerning the obtained differences with the simulations of the numerical model, inferior results are obtained for the FCM, GK, SGK and GG models for both time series. The best results are obtained for the SGG and MGG models for the first time series, and for the MGG model for the second time series. Simulation results for the SGG model, for the first time series, and for the MGG model for the second time series (data not shown) show a very close agreement between the simulation results of the numerical and the fuzzy model.

Figs. 9 and 10 display moisture profiles obtained with the fuzzy and the numerical model. The profiles for the first time series are obtained with the SGG model. A constant
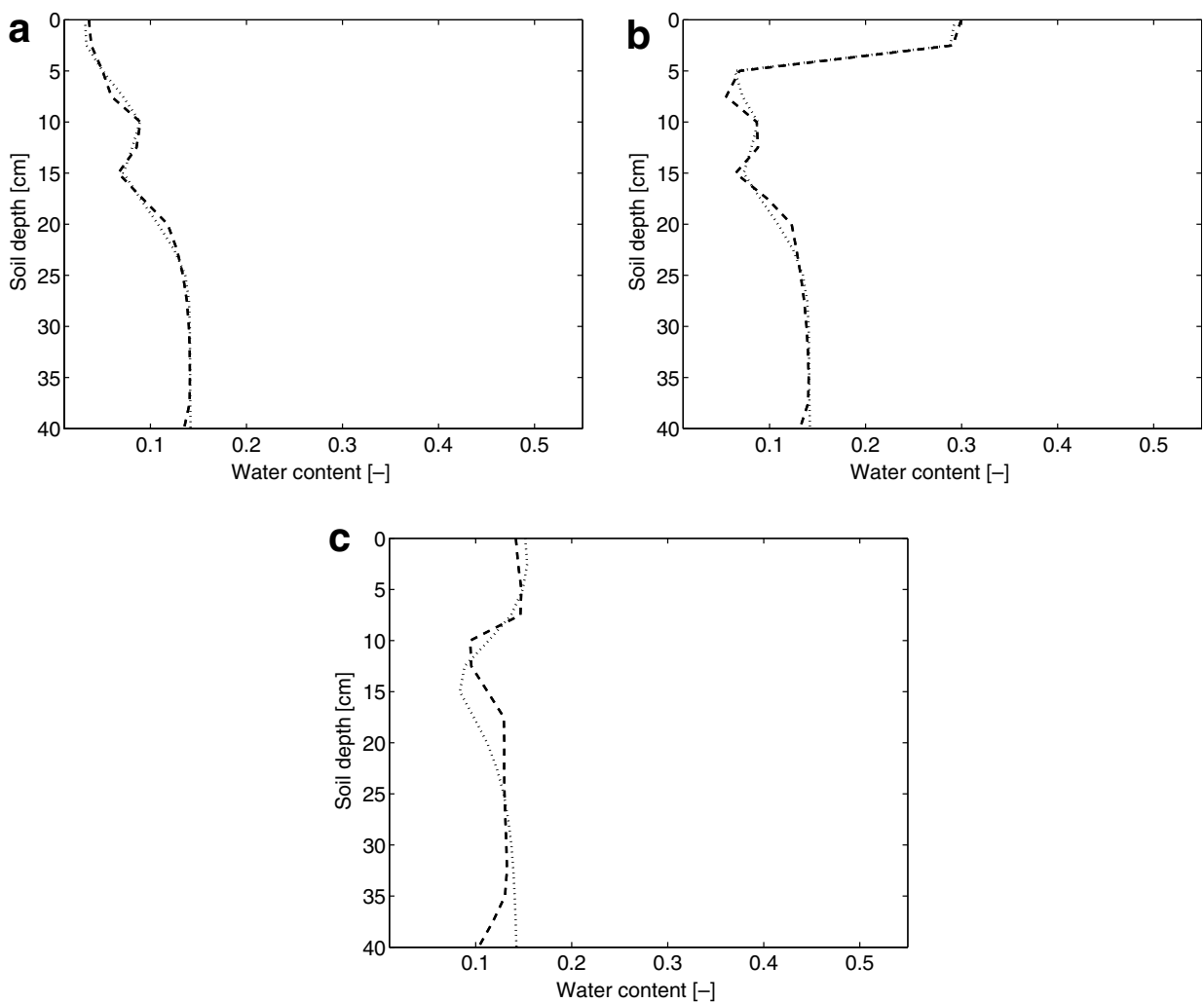

Fig. 9. Moisture profiles for the first time series. Results of the SGG model with $\beta=3$ (dashed line) and the numerical model (dotted line). The initial and lower boundary conditions used consist of interpolated water contents and a zero flux at the bottom of the profile. The time steps upon which these profiles are captured are indicated in Fig. 3. 

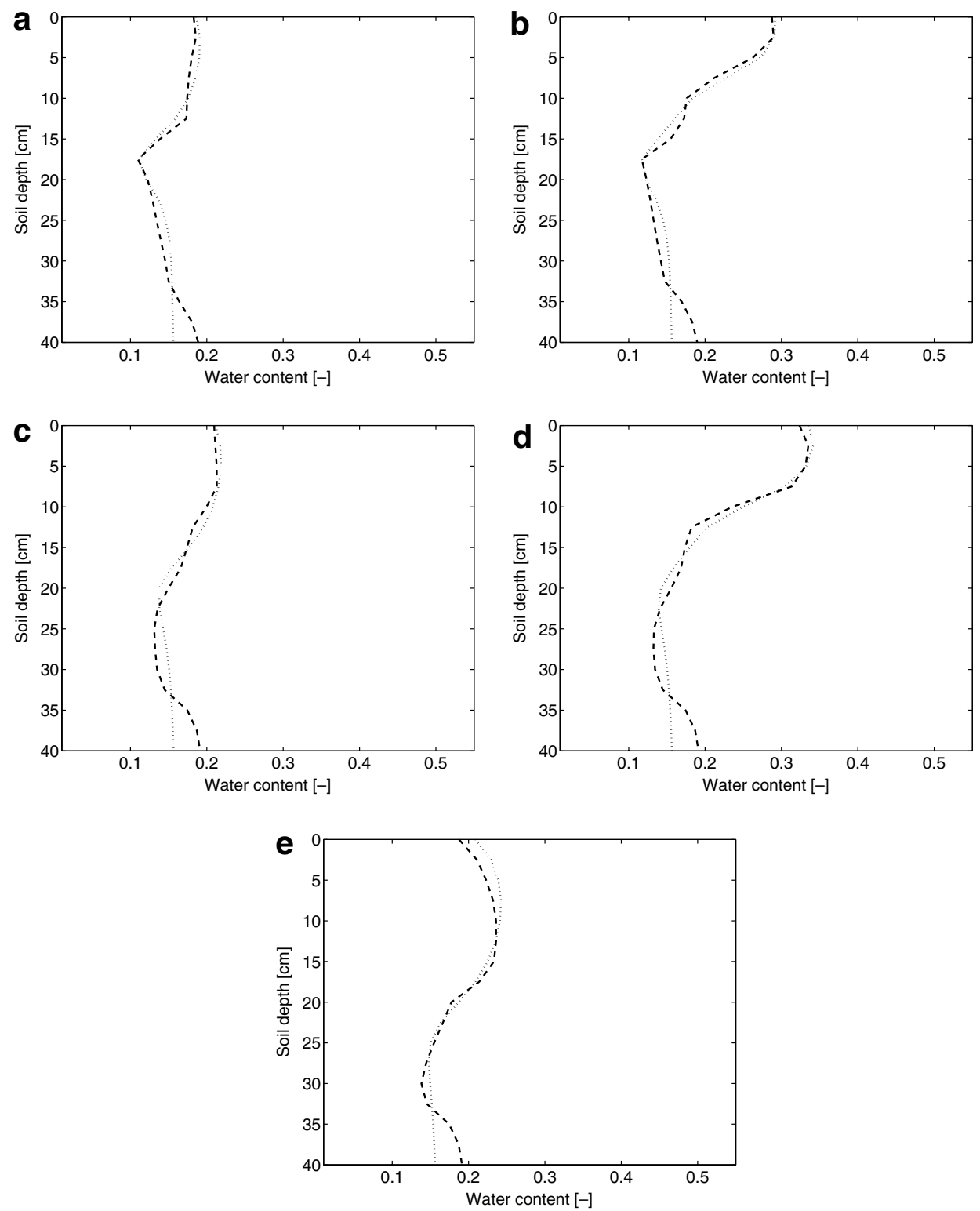

Fig. 10. Moisture profiles for the second time series. Results of the MGG model with $\beta=1$ (dashed line) and the numerical model (dotted line). The initial and lower boundary conditions used consist of homogeneous blocks of water content and a zero flux at the bottom of the profile. The time steps upon which these profiles are captured are indicated in Fig. 4.

zero flux was imposed at the bottom of the profile and the initial moisture conditions were obtained through interpolations of the measured water contents for depths between the measurement depths. The profiles for the second time series are obtained with the MGG model. A constant zero flux was also imposed at the bottom of the profile and initial conditions existed of homogeneous blocks of the measured water contents. The respective values of $\beta$ used for these models are 3 and 1 . A relatively good agreement between the different profiles is observed. Due to the accumulation of errors, the deviation between the profiles becomes larger as the simulation time proceeds, which is observed for both time series in the lower and central part of the profiles.
Since the two time series reach low and moderate water contents, the additional tests consisting of an increase of the initial water contents with $0.1,0.2$ or 0.3 were also performed on the SGG and MGG models, in order to evaluate the influence of the larger errors in the regions of higher water contents on the time series (see Tables 7 and 8). The models with their corresponding $\beta$-values that generally yielded best results on the original time series were selected and used to perform the additional tests. A similar behaviour as for models with multi-dimensional membership functions is noticed. The accuracy of the models decreases when high initial water contents are imposed (initial water contents are incremented by 0.3 ). Still, generally acceptable errors are obtained. However, depending on the 
Table 7

Mean absolute errors with the numerical model for the additional tests on the first time series

\begin{tabular}{|c|c|c|c|c|c|}
\hline \multirow[t]{2}{*}{ Clustering algorithm } & \multirow[t]{2}{*}{$\beta$} & \multicolumn{2}{|c|}{ Interpolation } & \multicolumn{2}{|c|}{$\begin{array}{l}\text { Homogeneous } \\
\text { blocks }\end{array}$} \\
\hline & & $\mathrm{ZF}$ & $\mathrm{CW}$ & $\mathrm{ZF}$ & $\mathrm{CW}$ \\
\hline \multicolumn{6}{|l|}{ Initial condition +0.1} \\
\hline SGG & 3 & 0.0105 & 0.0106 & 0.0114 & 0.0111 \\
\hline MGG & 7 & 0.0072 & 0.0079 & 0.0073 & 0.0061 \\
\hline \multicolumn{6}{|l|}{ Initial condition +0.2} \\
\hline SGG & 3 & 0.0048 & 0.0068 & 0.0058 & 0.0074 \\
\hline MGG & 7 & 0.0161 & 0.0183 & 0.0153 & 0.0177 \\
\hline \multicolumn{6}{|l|}{ Initial condition +0.3} \\
\hline SGG & 3 & 0.0147 & 0.0231 & 0.0138 & 0.0217 \\
\hline MGG & 7 & 0.0087 & 0.0149 & 0.0087 & 0.0150 \\
\hline
\end{tabular}

Table 8

Mean absolute errors with the numerical model for the additional tests on the second time series

\begin{tabular}{|c|c|c|c|c|c|}
\hline \multirow[t]{2}{*}{ Clustering algorithm } & \multirow[t]{2}{*}{$\beta$} & \multicolumn{2}{|c|}{ Interpolation } & \multicolumn{2}{|c|}{$\begin{array}{l}\text { Homogeneous } \\
\text { blocks }\end{array}$} \\
\hline & & $\mathrm{ZF}$ & $\mathrm{CW}$ & $\mathrm{ZF}$ & $\mathrm{CW}$ \\
\hline \multicolumn{6}{|l|}{ Initial condition +0.1} \\
\hline SGG & 3 & 0.0029 & 0.0035 & 0.0030 & 0.0035 \\
\hline MGG & 7 & 0.0088 & 0.0110 & 0.0083 & 0.0103 \\
\hline \multicolumn{6}{|l|}{ Initial condition +0.2} \\
\hline SGG & 3 & 0.0059 & 0.0058 & 0.0064 & 0.0052 \\
\hline MGG & 7 & 0.0135 & 0.0293 & 0.0129 & 0.0293 \\
\hline \multicolumn{6}{|l|}{ Initial condition +0.3} \\
\hline SGG & 3 & 0.0343 & 0.0313 & 0.0360 & 0.0312 \\
\hline MGG & 7 & 0.0204 & 0.0163 & 0.0220 & 0.0163 \\
\hline
\end{tabular}

initial and/or boundary conditions, different performances are observed. Concerning simulations with the models with a higher complexity, similar results as for the models with multi-dimensional membership functions are obtained.

\section{Conclusion}

In this paper, different methods were used to determine the antecedent parameters of TS models. The obtained models were incorporated into a fuzzy 1D groundwater model for the unsaturated zone. Results of an experiment carried out at the European Microwave Signature Laboratory were used to evaluate the performance of the fuzzy model, together with the results obtained by the numerical groundwater model of Hoeben and Troch [2]. Since the TS models were identified on a training surface obtained through the solution of the Darcy equation for a discretisation of the entire domain of water contents, more attention was given to the comparison of the results of both models than the comparison of the results with the measurements.

Evaluation of the error surfaces. It was observed that the error surfaces on the training data set showed higher errors when one of the two soil cells have a higher or saturated moisture content.
Evaluation of the simulation results. Concerning the simulation results on the time series, it was found that for models identified with the most popular algorithms, i.e. the fuzzy $c$-means and the Gustafson-Kessel algorithm, yielded inferior results compared to the models identified with the Gath-Geva algorithm and its variants. These latter models yielded very good results. For these models, results were very similar when the clusters were projected onto the input variable axes.

Evaluation of the soil profiles. Soil profiles were given to illustrate the behaviour of the models throughout the depth of the profile. The profiles obtained for the models with multi- and one-dimensional membership functions showed similar results. A good correspondence between the profiles of the fuzzy model and the numerical model was observed.

Evaluation of the additional tests. Since the experimental time series reach low and moderate water contents, the influence of the higher errors at the borders of the error surfaces could not be verified throughout these simulations. Therefore, some artificial additional tests were performed. The results were verified against the results obtained by the numerical groundwater model under the same conditions. In general, acceptable results were still obtained. The fact that the errors generally remain low, compared to the presence of higher errors in the error surfaces, is due to the pathways of water content combinations followed during wetting and drying phases in a soil profile. It was found that these pathways stay around the diagonal going from $(0,0)$ to $(1,1)$. Only under some initial and boundary conditions, some points of the pathway deviate from the diagonal.

Overall evaluation. Despite the presence of high errors at the borders of the training data set where one of the two soil cells reaches a high to saturated water content, the performance of the fuzzy groundwater model based on TS models with an optimal number of rules was relatively good. Although acceptable results are obtained, and more complex models are able to reduce the higher errors on the borders of high water content values, further research will verify whether or not simple models can be improved if they are identified on a training data set for which more data points are placed along the steep slopes of the training surface.

\section{Acknowledgement}

This research was funded by BOF-UGent, the special Research Fund of Ghent University, under Contract No. B/00066.

\section{References}

[1] Mancini M, Hoeben R, Troch PA. Multifrequency radar observations of bare surface soil moisture content: a laboratory experiment. Water Resour Res 1999;35(6):1827-38.

[2] Hoeben R, Troch PA. Assimilation of active microwave observation data for soil moisture profile estimation. Water Resour Res 2000;36(10):2805-19. 
[3] Celia MA, Bouloutas ET, Zarba RL. A general mass-conservative numerical solution for the unsaturated flow equation. Water Resour Res 1990;26(7):1483-96.

[4] Bárdossy A, Bronstert A, Merz B. 1-, 2- and 3-dimensional modeling of water movement in the unsaturated soil matrix using a fuzzy approach. Adv Water Resour 1995;18(4):237-51.

[5] Bárdossy A, Duckstein L. Fuzzy rule-based modeling with applications to geophysical, biological and engineering systems. New York, USA: CRC Press; 1995.

[6] Bárdossy A, Disse M. Fuzzy rule-based models for infiltration. Water Resour Res 1993;29(2):373-82.

[7] Babuška R. Fuzzy modeling for control, international series in intelligent technologies. Boston, USA: Kluwer Academic Publishers; 1998.

[8] Takagi T, Sugeno M. Fuzzy identification of systems and its applications to modeling and control. IEEE Trans Syst Man Cybern 1985;15(1):116-31.

[9] Vernieuwe H, De Baets B, Verhoest NEC. Comparison of clustering algorithms in the identification of Takagi-Sugeno models: a hydrological case study. Fuzzy Sets Syst 2006;157(21):2876-96.

[10] Gustafson D, Kessel W. Fuzzy clustering with a fuzzy covariance matrix. In: Proceedings of IEEE CDC, San Diego, CA, USA, 1979. p. 761-6.

[11] Höppner F, Klawonn F, Kruse R, Runkler T. Fuzzy cluster analysis. New York: John Wiley and Sons; 1999.

[12] Gath I, Geva AB. Unsupervised optimal fuzzy clustering. IEEE Trans Pattern Anal Mach Intell 1989;11:773-81.
[13] Abonyi J, Babuška R, Szeifert F. Modified Gath-Geva fuzzy clustering for identification of Takagi-Sugeno fuzzy models. IEEE Trans Syst Man Cybern Part B-Cybern 2002;32(5):612-21.

[14] Babuška R. Fuzzy identification toolbox for MATLAB, 2004. Available from: http://Icewww.et.tudelft.nl/ babuska/.

[15] Aström K, Wittenmark B. Computer controlled systems: theory and design. Englewood Cliffs, NJ: Prentice-Hall; 1984.

[16] Nash J, Sutcliffe J. River flow forecasting through conceptual models, Part $\mathrm{i}-\mathrm{a}$ discussion of principles. J Hydrol 1970;10:282-90.

[17] Beven KJ. Rainfall-runoff modelling, The primer. Chichester, UK: John Wiley and Sons; 2000.

[18] Hadjili ML, Wertz V. Takagi-Sugeno fuzzy modeling incorporating input variables selection. IEEE Trans Fuzzy Syst 2002;10(6):728-42.

[19] Xie X, Beni G. A validity measure for fuzzy clustering. IEEE Trans Pattern Anal Mach Intell 1991;13(8):841-7.

[20] Bezdek J. Pattern recognition with fuzzy objective function. New York: Plenum Press; 1981.

[21] Burrough PA, van Gaans P, MacMillan RA. High-resolution landform classification using fuzzy k-means. Fuzzy Sets Syst 2000;113(1):37-52.

[22] Richards L. Capillary conduction of liquids through porous media. Physics 1931;1:318-33.

[23] van Genuchten M. A closed-form equation for predicting the hydraulic conductivity of unsaturated soils. Soil Sci Soc Am J 1980;44:892-8. 